\title{
Effect of channel confinement on wake dynamics and forced convective heat transfer past a blunt headed cylinder
}

\author{
Sonal Bhadauriya ${ }^{\mathrm{a}}$, Harshit Kapadia ${ }^{\mathrm{b}}$, Amaresh Dalal ${ }^{\mathrm{c}, *}$, Sandip Sarkar ${ }^{\mathrm{d}}$

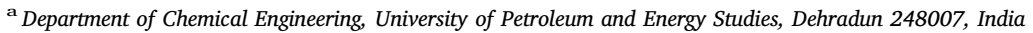 \\ ${ }^{\mathrm{b}}$ Department of Mechanical Engineering, Indian Institute of Science Bangalore, Bangalore 560012, India \\ ${ }^{c}$ Department of Mechanical Engineering, Indian Institute of Technology Guwahati, Guwahati 781 039, India \\ ${ }^{\mathrm{d}}$ Research and Development Division, Tata Steel Ltd., Jamshedpur 831007, India
}

\begin{abstract}
A B S T R A C T
In this paper, a two-dimensional numerical simulation is carried out to understand the effect of confinement (blockage ratio $\beta$ ) on fluid flow and forced convective heat transfer characteristics past a blunt headed cylinder. Utilizing air as an operating fluid, flow simulations are carried out for wide ranges of blockage ratios $(1 / 10 \leq \beta \leq 1 / 3)$ and Reynolds numbers $(60 \leq \mathrm{Re} \leq 200)$. The flow characteristics and heat transport are analysed critically for different $\beta$. The functional dependence of $C_{D}$ (Drag Coefficient) and $C_{L}$ (Lift Coefficient) on blockage ratio is examined. It has been found that $C_{D}$ reduces with increasing Re, while the Strouhal number and the average Nusselt number show an increasing trend when the blockage ratio is increased. The average Nusselt number also increases with increasing Re.
\end{abstract}

\section{Introduction}

Study of flow past two-dimensional bluff bodies and the resultant phenomena on vortex shedding has been the epicentre of various numerical and experimental investigations for decades. This kind of flow is encountered in many practical situations such as flow around suspension bridges, pillars, chimneys, masts etc. Due to their growing industrial importance, various other bluff body shapes are also paid a similar attention. These structures are used in electronic cooling equipment, cooling towers, heat exchanger tubes, offshore structures, and instrumentation equipment such as measurement probes and sensors. The oscillations of structures in a flow field are caused by the celebrated phenomena of vortex shedding. Sometimes these vibrations can lead to failure and damage when it matches with the natural frequency of the body during resonance. Therefore, it is critical to understand the fundamental physics behind this fluid-structure interaction mechanism not only for efficient designing but also in exploring the fundamental physics of occurrence.

In a channel (confined flow), the flow dynamics is highly affected by the blockage ratios $(\beta)$. Davis et al. [1] conducted both experimental and numerical study to investigate the effect of channel confinement on the average drag coefficient $\left(C_{D}\right)$ for a rectangular cylinder at various ranges of Reynolds numbers (Re). In a similar fashion, Suzuki et al. [2] performed numerical and experimental investigation for a channel obstructed by a square rod. They performed simulations for $37.5 \leq \operatorname{Re} \leq 150$ and the blockage ratios in the range $0.5 \geq \beta \geq 0.05$. They observed similar behaviour between the drag coefficient and the Reynolds number as reported by Davis et al. [1]. They also demonstrated a criss-cross motion of vortex shedding where the vortex shed from the upper half gradually moves towards the bottom wall and vice versa. Sohankar et al. [3] performed numerical simulations of flow around rectangular cylinders at low Reynolds number $(\operatorname{Re} \leq 200)$ and at a fixed blockage ratio 0.05 for different angle of incidences $(0 \leq \alpha \leq 90)$ and side ratios (1-4). Their study reported that the regime of the onset of vortex shedding is $50 \leq \mathrm{Re} \leq 55$ for side ratio of one and at $0^{\circ}$ angle of incidence. Breuer et al. [4] computed the two-dimensional laminar flow past a square cylinder $(\beta=1 / 8)$ for $0.5 \leq \operatorname{Re} \leq 300$ using two methods, namely, Lattice Boltzmann Automata (LBA) and Finite Volume Method (FVM). They found a reasonably good agreement between computed results utilizing each method. Abbassi et al. [5] conducted a numerical investigation of forced convection in a channel with a triangular prism at $20 \leq \mathrm{Re} \leq 250$ for air as an operating fluid. They found that the transition from symmetric flow to periodic flow occurs near $\mathrm{Re}=45$. Chakraborty et al. [6] investigated the wall effects for flow past a circular cylinder. They conducted simulations for a large range of Reynolds numbers $(0.1 \leq \mathrm{Re} \leq 200)$. They reported that for a fixed blockage ratio, both the angle of separation and length of recirculation zone increases with increasing Reynolds numbers. Sharma and Eswaran

\footnotetext{
* Corresponding author.

E-mail address: amaresh@iitg.ernet.in (A. Dalal).
} 


\begin{tabular}{|c|c|c|c|}
\hline \multicolumn{2}{|c|}{ Nomenclature } & $p$ & dimensionless pressure \\
\hline$D$ & projected diameter of the cylinder & $t$ & dimensionless time \\
\hline$H$ & height of the domain & $U_{\infty}$ & inlet velocity \\
\hline$L$ & length of the domain & & \\
\hline$C_{D}$ & coefficient of drag (dimensionless) & \multirow{2}{*}{\multicolumn{2}{|c|}{ Greek symbols }} \\
\hline$C_{L}$ & coefficient of Lift (dimensionless) & & \\
\hline$C_{L, r m s}$ & root mean square of the lift coefficient & $\tau_{i j}$ & dimensionless shear stress \\
\hline$F_{D}$ & drag force acting on the cylinder $(\mathrm{N} / \mathrm{m})$ & $\alpha$ & thermal expansion coefficient \\
\hline$F_{L}$ & lift force acting on the cylinder $(\mathrm{N} / \mathrm{m})$ & $\beta$ & blockage ratio $(\mathrm{D} / \mathrm{H})$ \\
\hline$L_{r}$ & recirculation length & $\gamma$ & thermal diffusivity of a fluid \\
\hline$\sigma_{\omega}^{L}$ & vorticity flux & $\theta$ & dimensionless temperature $\left(\theta=\frac{T-T_{\infty}}{T_{W}-T_{\infty}}\right)$ \\
\hline $\operatorname{Re}$ & Reynolds Number $\left(=\frac{\rho U_{\infty} D}{\mu}\right)$ & $v$ & Kinematic viscosity of a fluid \\
\hline $\operatorname{Pr}$ & Prandtl Number $\left(=\frac{\nu}{\gamma}\right)$ & $\begin{array}{l}\mu \\
\rho\end{array}$ & $\begin{array}{l}\text { Dynamic viscosity of a fluid } \\
\text { Density of a fluid }\end{array}$ \\
\hline St & Strouhal Number $\left(=\frac{g \alpha\left(T_{w}-T_{\infty}\right) D^{3}}{v^{2}}\right)$ & $\tau$ & time period for a complete cycle \\
\hline Gr & Grashof Number & $\omega$ & vorticity \\
\hline$R i$ & Richardson Number $\left(=\frac{G r}{\operatorname{Re}^{2}}\right)$ & \multirow{2}{*}{\multicolumn{2}{|c|}{ Subscripts }} \\
\hline$N u_{L}$ & local Nusselt Number (dimensionless) & & \\
\hline$N u_{\text {avg }}$ & average Nusselt Number over the cylinder surface & $R m s$ & root mean square value \\
\hline$u_{i}$ & dimensionless velocity in $i$-direction $\left(u_{i}={ }^{U_{i}} / U_{\infty}\right)$ & avg & average \\
\hline$U_{i}$ & dimensional velocity in $i$-direction & $w$ & wall \\
\hline $\begin{array}{l}x, y \\
X, Y\end{array}$ & $\begin{array}{l}\text { dimensionless Cartesian coordinates }\left(x=\frac{X}{D}, y=\frac{Y}{D}\right) \\
\text { dimensional Cartesian coordinates }\end{array}$ & $\infty$ & free stream condition \\
\hline
\end{tabular}

[7] studied the effect of channel confinement on the two dimensional laminar flow and heat transfer across a square cylinder for various blockage ratio and Reynolds numbers. They reported that as $\beta$ is increased, the channel wall first bestows stability to the flow until the critical value of $\beta$ is reached. Thereafter it imparts instability to the flow due to increased interaction between cylinder and the walls. Sahin and Owens [8] carried out a numerical study of wall effects up to high blockage ratio on two-dimensional flow past a circular cylinder. They investigated the effect on the critical Reynolds number for $\beta>0.7$ and its nature of instability.

Various numerical studies have focussed on confined flow past a square cylinder $[9,10$, and11]. All these studies report the inverse dependence of drag coefficient on Reynolds number. Dhiman et al. [10] also reported that the blockage ratio and Reynolds number influences the size of wake region and drag greatly compared to the power law index. Patil and Tiwari [12] analysed the effect of blockage ratio on wake transition for flow past square cylinders. They found that the onset of planar vortex shedding is delayed initially with the increase in blockage ratio. Sohankar [13] performed three-dimensional large eddy simulations of flow past rectangular cylinders and their side ratio effects on the wake dynamics. Kumar and Mittal [14] studied the effect of blockage on critical parameters for flow past a circular cylinder. They found that Strouhal Number increases with the blockage ratio. Mettu et al. [15] studied the dynamics of an asymmetrically placed circular cylinder in a channel. They reported that the amplitude of oscillations in the lift coefficient $\left(C_{L}\right)$ decreases as the blockage ratio increases. Singha and Sinhamahapatra [16] investigated the flow past a circular cylinder between parallel walls at low Reynolds number. They found that no vortex shedding occurs for flow Reynolds number of about 100 if normalized gap between the cylinder and walls in less than one.

De and Dalal [17] studied the confined flow past a triangular cylinder for a range of $80 \leq \operatorname{Re} \leq 200$ and blockage ratio $1 / 12 \leq \beta \leq 1 / 3$. They found at lower blockage ratios, flow is similar to the unconfined flow and is more prone to wake instability. Srikanth et al. [18] also performed simulations for a triangular cylinder placed in a channel. They found the critical Reynolds Number for onset of vortex shedding is nearer to 58-59. These studies $[17,18]$ also show the inverse dependence of drag coefficient on Reynolds number. Rosales et al. [19] performed simulations for inline and offset tandem pair of rectangular cylinders. They reported that the drag coefficient and Nusselt number decreases as the heated cylinder reaches the wall and the highest value of Strouhal number is observed in the channel centred, inline configuration.

In the literature reviewed, it can easily be found that studies on forced convective flow and heat transfer past a blunt headed bluff body are scare. Such geometry is very common now a day in electronic chips, large constructional frameworks such as pillars, columns and towers, etc. Therefore, this investigation is appealing in filling the gap in the literature. In addition, this geometry poses a complete new problem to work upon and generates a lot of academic curiosity. Our study primarily focuses on laminar unsteady flow and heat transfer past a confined blunt headed cylinder. The range of blockage ratio is considered as: $1 / 3 \geq \beta \geq 1 / 10$. The Reynolds number (based on projected length $\mathrm{D}$ ) range is from 60 to 200 , and the Prandtl number for the operating medium is 0.71 (air). Commercial CFD Solver Ansys Fluent (using noslip condition on channel walls and parabolic velocity profile for the

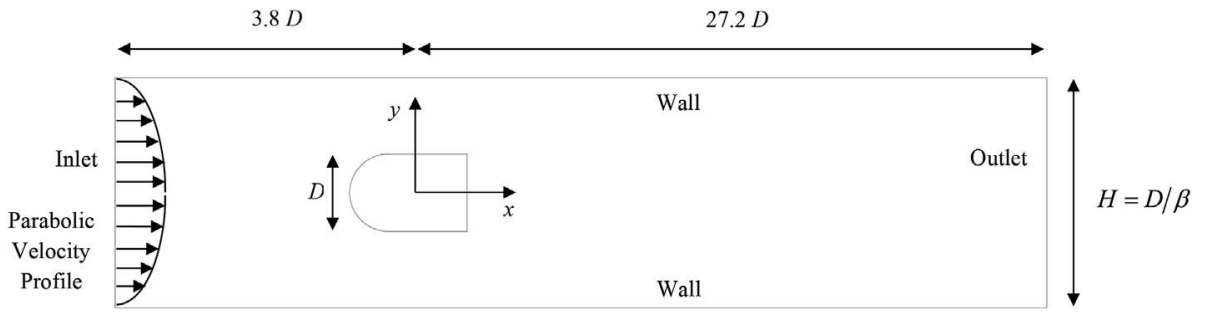

Fig. 1. Physical domain for computations. 
inlet) has been used as a simulation tool and computational mesh has been created using GAMBIT.

\section{Mathematical model}

As shown in Fig. 1, a blunt headed cylinder is placed in a channel with uniform cross flow at two dimensional flow regimes. Since the flow is along the axial $(X)$ direction, the gravity force acts along the transverse $(Y)$ direction. The geometry is composed of confluences between a square cylinder of dimension $D$ and a semi-circular cylinder of diameter $D$. The location of channel walls are fixed by the prescribed values of blockage ratio $\beta$ (ratio of the diameter of the cylinder to that of height of the domain, $\beta=D / H$ ). We explore the effect of channel confinement of the unsteady wake dynamic and heat transfer.

\subsection{Governing equations and boundary conditions}

The flow is assumed unsteady, two dimensional, laminar and incompressible with fluid properties remaining constant. The dimensionless governing equations for continuity, momentum, and energy are written in the following tensor form:

Continuity Equation:

$\frac{\partial u_{j}}{\partial x_{j}}=0$

Momentum Equation:

$\frac{\partial u_{j}}{\partial t}+\frac{\partial\left(u_{j} u_{k}\right)}{\partial x_{k}}=-\frac{\partial p}{\partial x_{j}}+\frac{1}{\operatorname{Re}} \frac{\partial^{2} u_{j}}{\partial x_{k} \partial x_{k}}$

Energy Equation:

$\frac{\partial \theta}{\partial t}+\frac{\partial\left(u_{k} \theta\right)}{\partial x_{k}}=\frac{1}{\operatorname{Re} \operatorname{Pr}} \frac{\partial^{2} \theta}{\partial x_{k} \partial x_{k}}$

where $\operatorname{Re}=\frac{\rho U_{\infty} D}{\mu}$ is the Reynolds Number and $\operatorname{Pr}=\frac{\nu}{\gamma}$, Prandtl Number. The fluid properties are described by the thermal diffusivity, $\gamma$ and the kinematic viscosity, $v$ As to symbols, $D$ is the length scale and $U_{\infty}$ is the velocity scale of the problem under consideration. $u_{i}$ is the nondimensional velocity along ith direction, $p$ is the dimensionless pressure, and $\theta$ is the dimensionless temperature.

The pertinent boundary conditions applied in the present simulation are as follows:

Inlet: A parabolic profile for the stream-wise velocity, Neumann condition for pressure, and a specified free stream temperature $\left(T_{\infty}\right)$ of the incoming fluid has been applied:

$u=1-4 \beta^{2} y^{2}$ for $\frac{-1}{2 \beta} \leq y \leq \frac{1}{2 \beta}$

$\frac{\partial p}{\partial x}=0$, and $\theta=0$.

Solid Boundaries: No-slip condition for velocity, normal gradient condition for pressure, and isothermal condition for temperature $\left(T_{W}\right)$ are used on the cylinder:

$u=v=0, \nabla p \cdot \hat{n}=0, \theta=1$

where $\hat{n}$ is the unit normal vector.

Channel Walls: At the channel walls, adiabatic $(\partial \theta / \partial y=0)$ condition for temperature, no-slip condition for velocity $(u=v=0)$, and zero Neumann condition of pressure $(\partial p / \partial y=0)$ is applied.

Outlet: The outflow boundary condition is used. The flow at the exit is close to fully developed condition and hence appropriate for the present set of simulations.

$\frac{\partial u}{\partial x}=0, \frac{\partial v}{\partial x}=0, \frac{\partial \theta}{\partial x}=0$

Drag, lift and moment coefficients:

The viscous and pressure forces acting on the cylinder are used to calculate drag, lift and moment coefficients. The drag coefficient is given by:

$C_{D}=\frac{F_{D}}{\frac{1}{2} \rho U_{\infty}^{2} D}$

where $F_{D}$ is the drag force acting on the cylinder.

The force exerted on the cylinder by the periodic fluctuations of flow is characterized by the lift coefficient and is given by:

$C_{L}=\frac{F_{L}}{\frac{1}{2} \rho U_{\infty}^{2} D}$

where $F_{L}$ is the lift force that acts on the cylinder in lateral direction.

The force coefficients are computed from the following expressions:

$C_{D}=2 \int_{S}\left(-p n_{x}+\tau_{x x} n_{x}+\tau_{x y} n_{y}\right) d S$

$C_{L}=2 \int_{S}\left(-p n_{y}+\tau_{x y} n_{x}+\tau_{y y} n_{y}\right) d S$

Here $p$ is non-dimensional pressure, $n_{x}, n_{y}$ are the $x$ - and $y$ components of unit normal vector to the surface $d S$. The integration is performed along the surface $(S)$ of the cylinder. The non-dimensional shear stress is given by:

$\tau_{i j}=\frac{1}{\operatorname{Re}}\left(\frac{\partial u_{i}}{\partial x_{j}}+\frac{\partial u_{j}}{\partial x_{i}}\right)$

\subsubsection{Strouhal number}

The frequency of vortex shedding characterizes the periodicity of the flow. The non-dimensional form of this frequency is known as Strouhal Number, and is defined as:

$S t=\frac{f D}{U_{\infty}}$

where $f$ is the vortex shedding frequency.

\subsubsection{Nusselt number}

Nusselt number characterizes the quantitative parameter of heat transfer. It quantifies the local heat transfer from the cylinder surface to the fluid. The non-dimensional form of Local Nusselt Number, $N u_{L}$ based on cylinder's projected diameter is defined as:

$N u_{L}=-\left.\frac{\partial \theta}{\partial n}\right|_{\text {along the cylinder surface }}$

where $n$ denotes the direction normal to the cylinder.

The local Nusselt number computed in this unsteady range is timeaveraged over several periods of vortex shedding cycle. The physical significance of the local Nusselt number variation is the variation of the local magnitude of heat transfer along the bluff body surface. The surface averaged value of the local Nusselt number is defined as the average Nusselt Number. The average Nusselt number has been calculated by time averaging the spatially averaged local Nusselt number over the cylinder surface. This is defined as:

$N u_{\text {avg }}=\frac{1}{L} \int_{L} N u_{L} d L$

A fixed two dimensional blunt headed cylinder with projected $D$ is placed in a channel with a parabolic velocity profile. The cylinder surface is kept at a constant temperature $T_{W}$. The flow is assumed incompressible with constant thermo physical properties. The domain length, $L=31 D$ and height, $H=D / \beta$, where, $\beta$ is the blockage ratio.

\subsection{Solution methodology}

The governing equations with the corresponding boundary conditions are solved using a finite volume method based solver ANSYS FLUENT [21]. The Semi Implicit Method for the Pressure Linked 
Equations (SIMPLE) algorithm is used to avoid pressure-velocity decoupling. The main importance of choosing SIMPLE algorithm is its stable convergence behaviour for unsteady problems. A third order upwind scheme QUICK has been used to discretize the convective terms in the momentum and energy equations. The unsteady solver is used for the time integration. A second order accurate Adams-Bashforth implicit scheme is used for time. The convergence criterion of $10^{-6}$ is used for the residuals of continuity, $x$-momentum and $y$-momentum equations, whereas a criterion of $10^{-10}$ is used for the residuals of the energy equation. To input the physical properties of the fluid the constant density method was used. It should be mentioned here that these values of the input parameters are insignificant in the solution as the final results are presented in non-dimensional form. The unsteady calculations keep on running till the system attains its dynamic steady state condition. Such dynamic steady state condition in the present study is said to be achieved when the transient behaviours of coefficient of lift and average Nusselt number are observed to reach saturation (i.e. the values of those properties fluctuate with a constant frequency and amplitude with successive time steps). To prevent numerical oscillations, the necessary conditions for time stepping are determined from the Courant-Friedrichs-Lewy (CFL) condition. The required time step is taken by satisfying CFL condition at all-time marching steps. A nondimensional time step size $(\Delta t)$ of 0.003 is used satisfying the above restrictions. It is noticed that with increasing values of Richardson number there is a considerable increase in computational time for achieving the same level of convergence $[20,21]$.

\subsection{Grid generation and code validation}

The number of grid points and their distribution also influence the parameters to a huge extent. This happens due to the complexity of the physical process associated with vortex shedding and flow separation. The solution accuracy also depends upon the structure and architecture of the domain. A non-uniform grid with finer grid distribution near the cylinder discretizes the computational domain. It is done to capture the boundary layer separation and vortex street more precisely. The hybrid grid is created using the commercial software GAMBIT [22]. Unstructured triangular mesh is used near the semi-circular part of the body and it is used so that it captures the steep gradients near the cylinder surface. A closer view nearer to the cylinder is shown in Fig. 2.

To check the accuracy and reliability of the solution, present numerical code is validated by performing fluid flow and heat transfer simulations past a square cylinder at $0^{\circ}$ incidence at $\operatorname{Re}=100$, $\operatorname{Pr}=0.71$, and for various Richardson Numbers $(-1 \leq R i \leq 1)$. The results are obtained in terms of integral coefficients $\left(C_{D}, N u\right)$. Table 1 summarizes the comparison of presents results with the ones reported in the literature [20]. As can be seen the deviation between the present data and literature reviewed is very less. The present results are in excellent agreement with the literature [20]. To ascertain further confidence in the current computational model, we further compare our existing simulation results with those reported in the literature [7]. The predicted results by the present numerical model and with the existing results reported in literature [7] are also appended in Table 1. The comparison reveals excellent agreement between the present results with those of literature [7]. In addition, to check the validity of our numerical results with the available experimental results, we have performed computations for the forced convective flows past a circular cylinder in cross stream for various ranges of Reynolds numbers. Fig. 3a compares the Strouhal number $(S t)$ variation with the experimental results due to Williamson [23]. Whereas, Fig. 3b shows the comparison results of local Nusselt number $\left(\mathrm{Nu} u_{l}\right)$ distribution over the cylinder surface with the experimental results of Eckert and Soehngen [24] for $\operatorname{Re}=218$. The comparison of our computed solution for the Strouhal number and local Nusselt number are in good agreement with those of the above published experimental results.

\subsection{Grid independence test}

A grid independence test is performed to analyse and optimize the accuracy and computational time required for the simulation. A sufficiently fine grid is required to capture flow structure precisely but a highly refined grid leads to higher computational costs. Grid independence test is carried out with respect to $C_{D}, C_{D(\text { Wall })}, S t$, and $N u_{\text {avg }}$, for $\beta=1 / 5, \operatorname{Re}=120$. Corresponding to the architecture of the present domain, four different meshes are created: M1, M2, M3, M4, and are tabulated in Table 2. $C_{N}$ corresponds to the number of finite volume cells over the cylinder. The results of the grid independence test have also been appended with Table 2 .

From Table 2, it is clearly understood that with meshes M3 and M4, the variations of different parameters show minimal percentage, e.g. $C_{D}$ shows $0.011 \%, C_{D(\text { Wall })}$ shows $0.007 \%, N u_{\text {avg }}$ shows $0.008 \%$ whereas $S t$ shows $0.51 \%$. Therefore, for the present computations the grid independent situation is attained for mesh M3. It is computationally economical for all different cases studied in the present investigation.

\section{Results and discussion}

The present investigation aims in analyzing the effect of blockage ratio, $\beta$ on the unsteady wake dynamics and heat transfer characteristics past a blunt headed cylinder placed in a channel in cross flow configuration. The ranges of blockage ratios considered in this study are $1 / 3 \geq \beta \geq 1 / 10$. Utilizing air as an operating media $(\operatorname{Pr}=0.71)$, the Reynolds number (based on projected length $D$ ) range is taken as $60 \leq \operatorname{Re} \leq 200$. The results obtained correspond to dynamic steady state conditions. To check whether the system has reached dynamic steady state, simulations have been performed for about 20 periodic cycles till the asymptotic vortex shedding frequency has been achieved.

\subsection{Flow and temperature fields}

The characteristic features of the wake past a bluff body is customarily visualized in terms of the contours of streamlines, vorticity, and temperature. Detailed physics of those typical features can be ascertained by examining wake structure interactions. Accordingly, Fig. 4a-c delineates the instantaneous contours of streamlines (Fig. 4a), vorticity (Fig. 4b), and isotherms (Fig. 4c) at a representative Reynolds number, $\operatorname{Re}=120$, and blockage ratios of $\beta=1 / 3,1 / 6$ respectively. Evidences of vortex shedding are apparent in Fig. 4a-c. It is noticed that the channel confinement effect becomes more predominant at higher value of blockage ratios, where the positive vortices forming at the channel walls start interacting with the negative vortices generated at the upper half of the cylinder and vice versa is the situation at the lower half. The migrated shear layers from the walls start merging with the shedded vortices from the cylinder, and thus increasing the strength of the primary vortex strand. This mechanism triggers in augmenting the strength of the shear layers generated at the channel walls and ultimately leading to an increased cross stream oscillation nearer to the

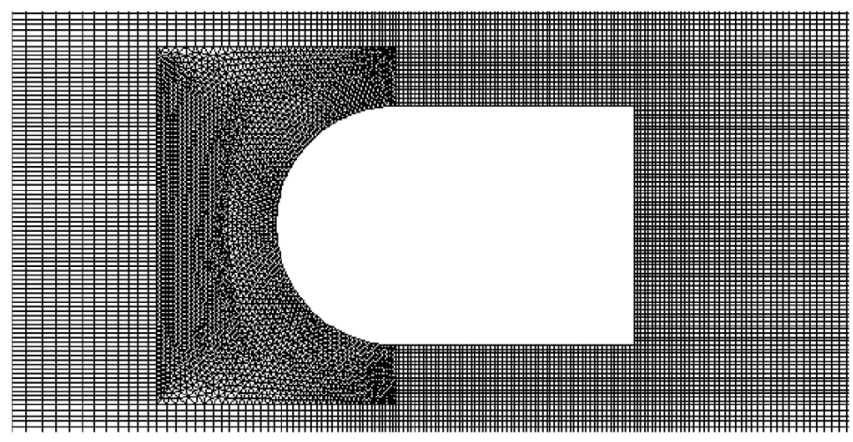

Fig. 2. A closer view of the grid around the cylinder. 
Table 1

Comparison of present flow results with literature for $\operatorname{Re}=100$ and $\operatorname{Pr}=0.71$.

\begin{tabular}{|c|c|c|c|c|c|c|}
\hline \multirow[t]{2}{*}{$\mathrm{Ri}$} & \multicolumn{2}{|c|}{ Dulhani et al. [20] } & \multicolumn{2}{|c|}{ Sharma and Eswaran [7] } & \multicolumn{2}{|c|}{ Present } \\
\hline & $\mathrm{Nu}$ & $C_{D}$ & $\mathrm{Nu}$ & $C_{D}$ & $\mathrm{Nu}$ & $C_{D}$ \\
\hline-1 & 4.32 & 1.78 & - & - & 4.33 & 1.77 \\
\hline-0.5 & 4.20 & 1.63 & - & - & 4.19 & 1.62 \\
\hline 0 & 4.10 & 1.56 & 4.1 & 1.5 & 4.08 & 1.55 \\
\hline 0.5 & 4.20 & 1.63 & - & - & 4.19 & 1.62 \\
\hline 1 & 4.32 & 1.78 & - & - & 4.33 & 1.77 \\
\hline
\end{tabular}

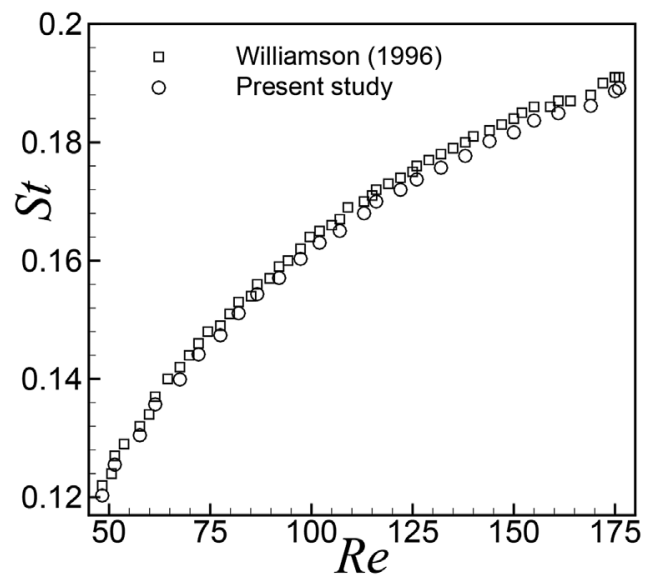

(a)

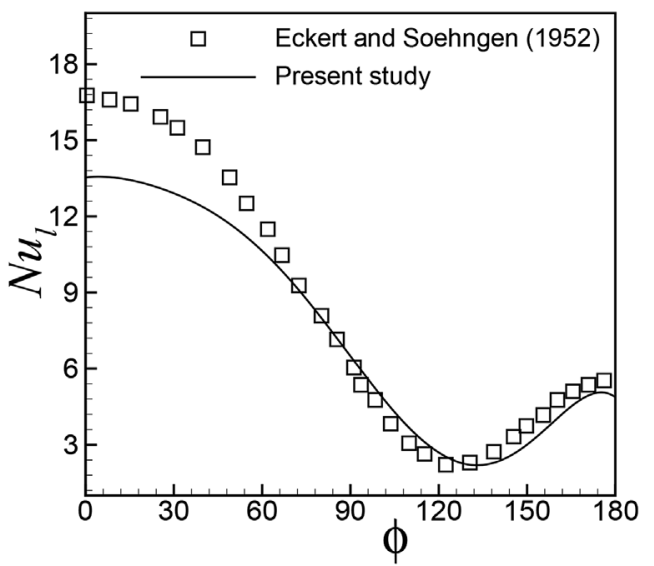

(b)

Fig. 3. (a) The Strouhal number $(S t)$ variation with the Reynolds number for forced convective flows past a circular cylinder. (b) The local Nusselt number $\left(\mathrm{Nu} \mathrm{u}_{l}\right)$ distribution over a circular cylinder in forced convection for $\operatorname{Re}=218$ (here $\phi$ is the polar angle).

Table 2

Results of grid independence test.

\begin{tabular}{lllllll}
\hline Mesh & Total No. of cells & $C_{N}$ & $C_{D}$ & $C_{D(\text { Wall })}$ & $N u_{\text {avg }}$ & $S t$ \\
\hline M1 & 60564 & 210 & 2.7020 & 1.42462 & 4.5813 & 0.1755 \\
M2 & 84914 & 240 & 2.7026 & 1.42466 & 4.5800 & 0.1748 \\
M3 & 111748 & 260 & 2.7033 & 1.42480 & 4.5800 & 0.1748 \\
M4 & 134532 & 280 & 2.7036 & 1.42470 & 4.5796 & 0.1739 \\
\hline
\end{tabular}

vicinity of the walls. As a result, flow starts to separate from the walls by forming recirculation bubbles, which finally convect in the downstream direction with the main flow. This may eventually lead to actuate the global flow instability, and therefore bringing early formation of three dimensional structures towards an instability in the flow. Contrary to $\beta=1 / 3$, a stable shearing motion of the vorticity dynamics is observed. Streamlines show smooth sailing over the blunt head and corresponding formations of center and saddle points after separating of the cylinder to the exit plane. A greater suppression in the recirculation zone is found at the higher blockage ratio in compared to the lower. This is due to the effect of transmission of the shearing momentum from the channel walls affecting the primary recirculation bubble. Furthermore, the streamline is somewhat stretched more toward the downstream direction at $\beta=1 / 3$. The isotherms show similar behaviour to that of vorticity. It has been stated in the literature that the vorticity magnitude always coincides with the magnitude of maximum temperature. However, a higher crowding of the isotherms at the rear stagnation zone is seen at $\beta=1 / 3$ compared to that of at $\beta=1 / 6$. This behaviour will eventually reflect in heat transfer rate as the rate of creeping in temperature lines is proportional to that of the magnitude of the temperature gradient at the cylinder surface, and thus controlling the heat transfer rate. Analogous to that of streamlines, the advection of temperature contours are stretched further downstream direction from the cylinder surface.

\subsection{Vortex shedding cycle}

Fig. 5a-b the instantaneous contours of streamlines (Fig. 5a) and isotherms (Fig. 5b) captured at successive instances of time instants during complete vortex shedding cycle. The results have been shown at fixed values of $\operatorname{Re}=150, \beta=1 / 3$. Typical instances of Karman vortex shedding can be noticed from Fig. 4. The vortex shedding process confirms to the successive stages as detailed in Biswas and Sarkar [25]. As evident from stream line plots, the growth of the shear layers emanating from each sides of the cylinder shoulder takes place asymmetrically at various phases of the vortex shedding. Alternate crossover motion of the re-circulatory coherent streamline blob can be seen during vortex shedding instances. The flow topology indicates the formation of multiple saddle points with changing locations during transient time instants of vortex shedding. The advection of thermal energy from the cylinder surface to the downstream takes place primarily by the vortices. Those vortices generate due to the roll-up of the shear layers during a set of interconnected time dependent stages. During advection of heat from the cylinder surface, a high temperature gradient forms at the vortex boundary. The vortex shedding cycle clearly manifests the criss-cross motion of the isotherms with increasing transverse extent of the plume at the downstream direction. The blob of hot fluid initially originated from the cylinder shoulder grows in size and finally separated from its own source due to the continuous accumulation and constriction process. The accumulation process increases the strength of the hot fluid blob, whereas the constriction process causes detachment from its own source due the progressive enhancement in shear rate. It is worth noting here that the in general the end of the recirculation bubble coincides with the end of the formation zone of the strand of the temperature [26].

\subsection{Time averaged fields}

The time-averaged field is obtained by time averaging the instantaneous fields of velocity, and temperatures over successive instants of vortex shedding cycle. The field samples are collected after the system has reached dynamic steady state. At a fixed Reynolds number of $\mathrm{Re}=180$, Fig. 6(a)-(c) display the time-averaged contours of streamlines (Fig. 6a), vorticity (Fig. 6b), and isotherms (Fig. 6c), at two representative values of blockage ratios, namely, $\beta=1 / 3$ and $1 / 6$.

From the time-averaged plots, it is seen that the time-averaged streamlines show reflection symmetry with respect to the channel centreline. The strength of the recirculation zone at $\beta=1 / 6$ is found to be higher than that of at $\beta=1 / 3$. The separated shear layers emanating from the cylinder vertices form a thinner neck that joins with the tip of the primary coherent vortex blob and causes lesser stretching of the vortices. Streamline curvature for the global flow is evident at the near 


$$
\beta=1 / 3
$$

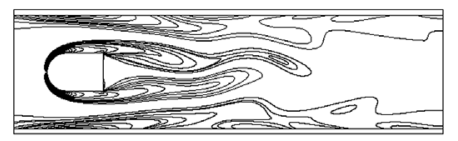

$$
\beta=1 / 6
$$

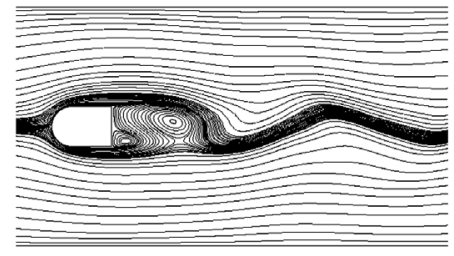

(a)

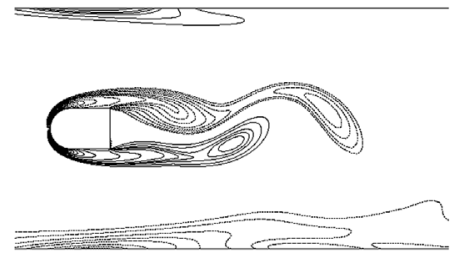

(b)
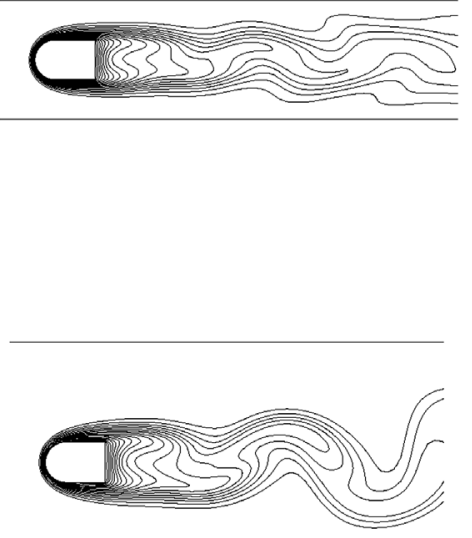

(c)

Fig. 4. Instantaneous contours of (a) streamlines, (b) vorticity, and (c) isotherms, at a representative Reynolds number, Re $=120$, and blockage ratios of $\beta=1 / 3$, $1 / 6$.
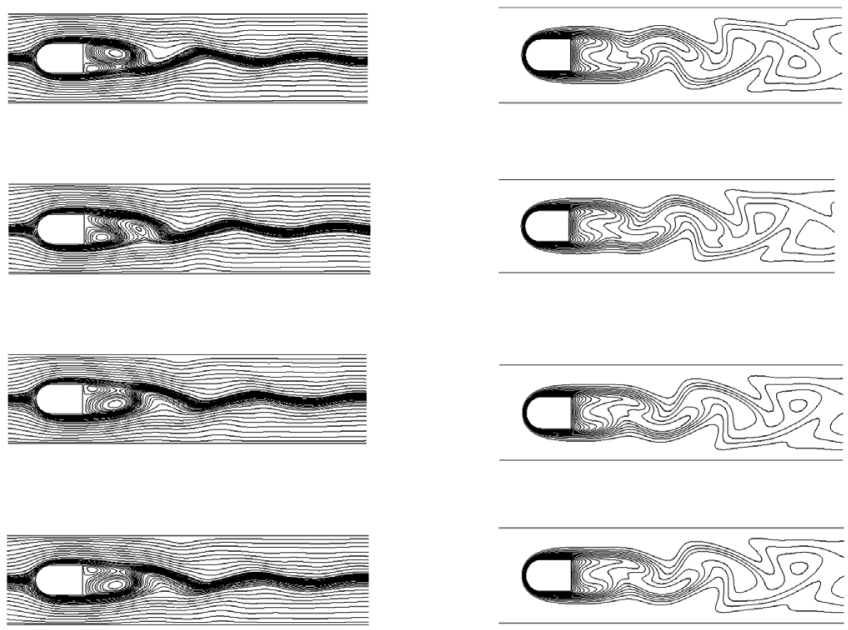

(a)

(b)

Fig. 5. Instantaneous contours of (a) streamlines and (b) isotherms captured at successive instances of time instants during complete vortex shedding cycle at $\operatorname{Re}=150, \beta=1 / 3$.

channel wall region for $\beta=1 / 3$. This is because of the fact that at higher values of blockage ratios the boundary layer fluid motion is hampered due to the continuous interactions of the wall generated shear layer with that of bulk flow. This causes generation of additional momentum along the transverse direction of the flow, this gradually transmitted to the primary fluid motion, and thereby initiation of flow constriction effect. Such phenomena eventually amplify the magnitude of the flow velocity along the axial direction. The time averaged vorticity contours show symmetric distributions for all values of blockage ratios. The overall trend of distribution follows origination of positive and negative vortex strand at the forward stagnation point. The tip of the strand shifts its location as the channel confinement it reduced. At the transition zone (from the curve to the straight edge) no instances of flow separation is noticed. Furthermore, at $\beta=1 / 3$ the vortices near the walls are much more prominent as compared to the lower confinement, $\beta=1 / 6$. This is due the enhanced strength of the wall vorticity strands at higher values of $\beta$. The time averaged isotherms also show symmetric distributions about the symmetry line. There is a structural similarity between the isocontours of vorticity and temperatures, since they share the same source of origination. Isotherms are found to be creeping at the right and left edges of the cylinder, thus indicating higher Nusselt number and thinning of thermal boundary layers, which finally separates from the rear-end vertices. For the higher confinement $\beta=1 / 3$ the isotherm are spread in a broader fashion as compared to that for a lower confinement $\beta=1 / 6$.

\subsection{Force coefficients and Strouhal number}

As a result of the fluid flow, there are forces acting on the cylinder, namely, the drag force acting in the stream wise direction and lift force acting in the lateral direction. The resultant forces subjected to the cylinder are arising from the normal and shearing stresses along the lateral and axial directions respectively. These cylinder forces (drag and lift) fluctuate periodically and are computed after the flow reached dynamic steady-state situation. This happens due to periodic vortex shedding alternatively from both upper and lower half of the cylinder. The integral coefficients, namely drag $\left(C_{D}\right)$, and $r m s$ values of the lift coefficient $\left(C_{L, r m s}\right)$ are obtained by time averaging the periodic signals of drag, and rms values of lift coefficients. Fig. 7a-b represents the variations of $C_{D}$ (Fig. 7a), and $C_{L, r m s}$ (Fig. 7b) with Re for different values of $\beta$. The behaviour of $C_{D}$ with Re for various $\beta$ (Fig. 7a) exhibit various characteristic features upon analysis. The force required to put the cylinder in motion in an incipient media is called time averaged drag coefficient, $C_{D}$. The drag coefficient $C_{D}$ is inversely proportional to Re. As Reynolds Number is increased, the drag coefficient is decreasing. Square and rectangular cylinders do not show this inverse dependence. This behaviour is reported for circular cylinders [6]. As the confinement increases, the value of drag also increases. Thus, Drag coefficient is directly proportional to the blockage ratio. As we increased the blockage from $10 \%$ to $33.33 \%$, the value of drag coefficient doubles itself for $\mathrm{Re}=60$ (Fig. 7a). We can also observe that as the confinement height is decreased, the dependence of $C_{D}$ on Re also becomes less pronounced. For the ranges of the higher values of Reynolds number $(\operatorname{Re}=180,200)$ and low confinements, the values of drag coefficients are approximately similar. Thus, for a constant blockage ratio the drag 


$$
\beta=1 / 3
$$
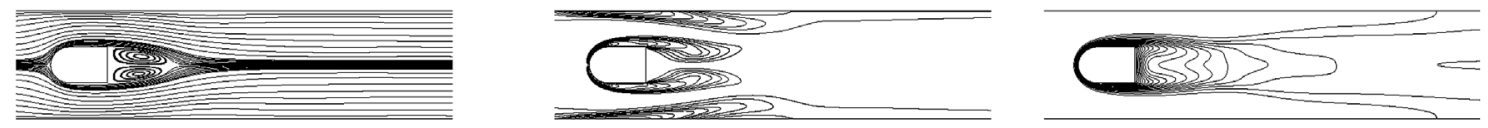

$$
\beta=1 / 6
$$
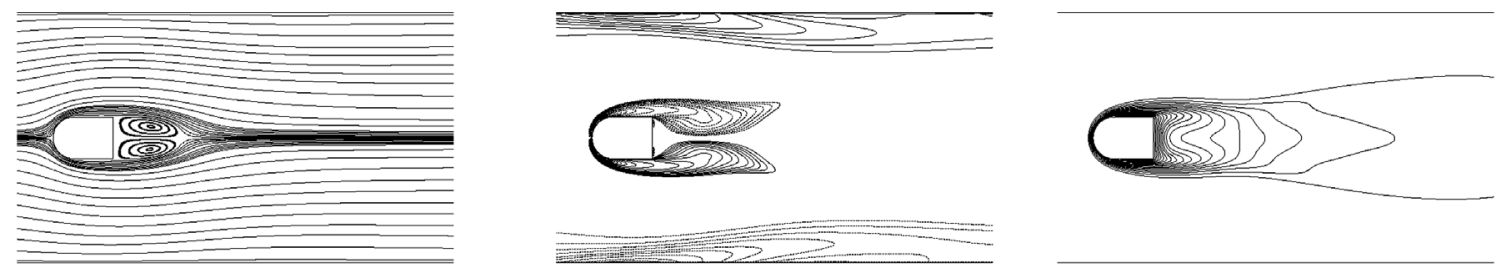

(a)

(b)

(c)

Fig. 6. Time-averaged contours of (a) streamlines, (b) vorticity, and (c) temperature, at $\operatorname{Re}=180, \beta=1 / 3$ and $1 / 6$.

force acting on the wall is inversely proportional to the Reynolds number. The behaviour of $C_{L, r m s}$ is shown in Fig. 7b. It is observed that for a constant value of Re, the value of $C_{L, r m s}$ increases with decrease in confinement. As the blockage ratio is increased, the value of $C_{L, r m s}$ decreases. However, for higher confinements as seen for the case of $\beta=1 / 3$, the value of $C_{L, r m s}$ was observed to be higher than that of $\beta=1 / 4$, and $1 / 5$, as the Reynolds number is increased above 120 . This happens due to the increase in the disturbance created by the channel walls. For a constant blockage ratio, the value of $C_{L, r m s}$ increased with the increase in Re.

\subsubsection{Strouhal number}

The Strouhal number is calculated by taking the Fast Fourier Transform (FFT) of the temporal evolution of lift signal to saturation. The highest peak of the harmonics in the FFT graph depicts the corresponding Strouhal number in the flow. Physically, Strouhal number indicates the frequency of the vortex shedding. The variation of St with Re for various $\beta$ is shown in Fig. 8. It is found out that for a fixed value of Re, the magnitude of $S t$ increases with increasing $\beta$. For a constant $\beta$, the value of Strouhal number shows its classical trend by increasing its magnitude with augmenting the value of Re. Furthermore, for $\beta \leq \frac{1}{8}$, the functional variation in Strouhal number curve with lowering the value of $\beta$ become progressively smaller. This typical behaviour clearly manifests that the frequency of vortex shedding increases at the higher Strouhal number value. This causes in amplifying the rate at which the coherent vertical structures start pinched off from the cylinder during consecutive instants of vortex shedding cycle. As a result, the shear layer become destabilized with the continuous interactions from the channel wall generated shear layers. This phenomenon becomes more prominent at the higher wall confinements. The overall wake dynamics become more complex with the occurrences of multiple large and small scale vortex structures. Similar behaviour is also reported by earlier researchers $[7,14,17]$.

\subsection{Recirculation length}

Recirculation length is defined as the lateral distance from the back stagnation point to the wake stagnation point of a bluff body. For unsteady flow regime, it is calculated from the the time averaged contours of streamlines forming re circulation loop. Fig. 9 shows the variation of recirculation length, $L_{r}$ as a function of blockage ratios $\beta$, for two representative cases of steady $(\mathrm{Re}=60)$ and unsteady $(\mathrm{Re}=150)$ flow situations. During the steady case at $\operatorname{Re}=60$, the length of the recirculation zone decreases with increasing the value of $\beta$. The corresponding physical explanations may be offered as follows: during the steady case, the zone of separation progressively suppresses owing to the continuous enhancement of flow velocity passing through the passage between top and bottom half of the cylinder shoulder and the channel walls. This brings an additional momentum acting nearer to the separation points and thus shifting its position. The resultant influence is to traverse the wake stagnation point towards the back stagnation point and as a result reducing its overall length. Such effects are lesser at the higher values of Reynolds number. At the higher values of Re, the inertia effect become predominant, this, in turn, reduces the magnitude of that additional momentum to a substantial degree. Additionally, for such unsteady regime, the periodicity in the flow alters the flow dynamics altogether, where the wake structure interactions plays a major role in altering the separation zone at the rear stagnation region. It is worth mentioning here that for higher values of Reynolds numbers, the variation in recirculation length is marginal when the blockage ratios are progressively enhanced.

\subsection{Vorticity flux}

The vorticity flux for various Reynolds numbers has been calculated at a representative location of $x=2$ (where, $x$ is the dimensionless horizontal linear distance away from the inlet of the channel; $x=X / D$, $X$ being the dimensional distance). The local time-averaged vorticity flux is defined at the vertical cross section, and at $x=2$ as [26]:

$\left.\sigma_{\omega}^{L}(y)\right|_{x=2}=\left.\frac{1}{\tau} \int_{0}^{\tau} u \omega(y, t)\right|_{x=2} d t$

Where, $\tau$ is the time period for a complete cycle and $\omega$ is defined as the vorticity. The parameter $\sigma_{\omega}^{L}$ is the quantity that captures the vorticity generated upstream of the cross section $x=2$ during vortex shedding cycle. Fig. 10a shows the variation of $\sigma^{L}{ }_{\omega}$ along the vertical cross section for various values of Reynolds numbers and at $x=2$, $\beta=1 / 3$. The results have been shown for various values of Reynolds numbers. It can be deciphered from the figure that the general trend of the vorticity magnitude is found to be maximum near the walls. The magnitude of vorticity flux increases with the increase in Re [26-28]. The functional behaviour of the $\sigma^{L}{ }_{\omega}$ variation follows similar profile (for every Re), this qualitatively indicates that the vortex formation and shedding is taking place in the same manner irrespective of the 


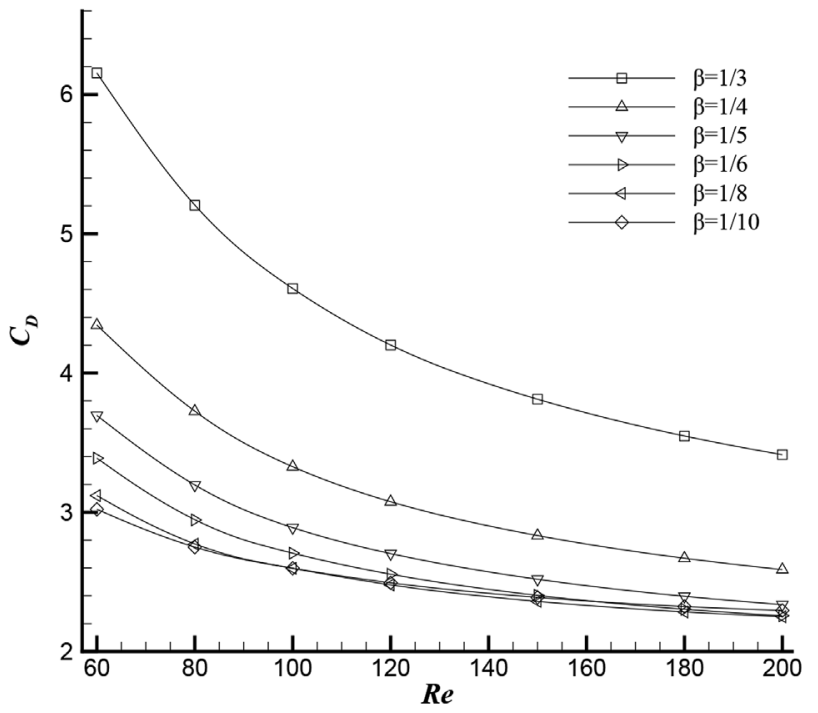

(a)

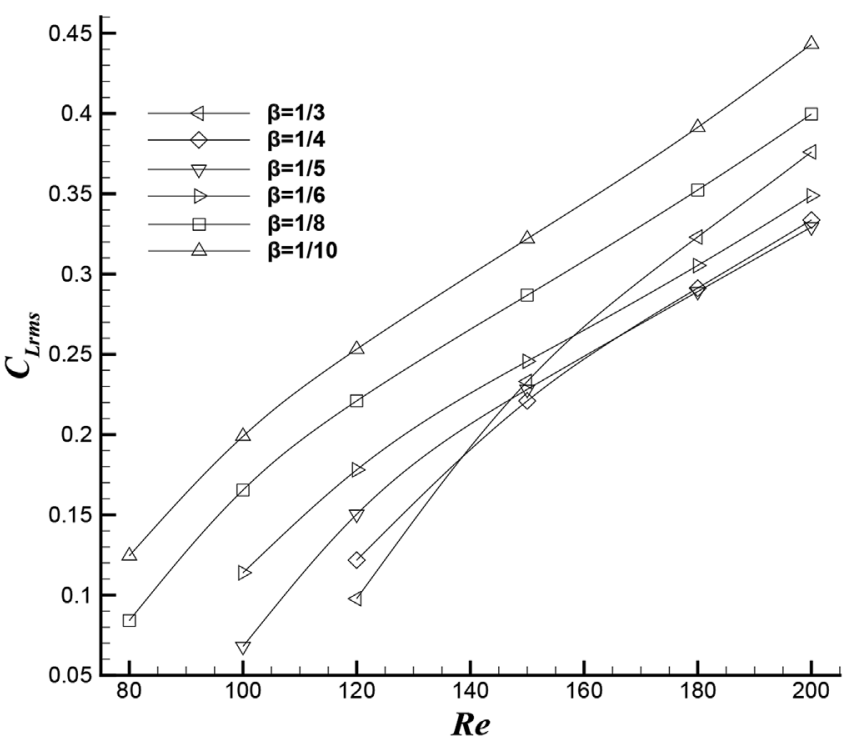

(b)

Fig. 7. The variations of (a) $C_{D}$, and (b) $C_{L, r m s}$ with Re for different values of $\beta$.

Reynolds number, the difference comes in changing the global magnitude. At the mid-plane of the domain, the vorticity flux is zero as clearly seen from the graph. In addition, the vortices produced at equal distance from the origin have the same magnitude but with opposite signs. Similar behaviour is evident for all values of the Reynolds numbers. The maximum vorticity magnitude is always shown near to the walls of the channels.

Fig. 10b depicts the variation of $\sigma^{L}{ }_{\omega}$ across various cross sectional planes for $\mathrm{Re}=150$. It is observed that the vorticity magnitude is maximum at the cross section which is nearer to the cylinder. At $x=1$, the maximum value of vorticity magnitude is shown. As we move away from the body, this magnitude starts decreasing. For $x \geq 10$, the variation in the flux is negligible. Again, the maximum vorticity magnitude is seen near to the channel walls. Furthermore, the magnitude of the vorticity flux at the same distance from the origin is equal but opposite in signs. As one moves away from the body, the peaks start flattening, which implies as one moves away, the reduction in vorticity magnitude is much more gradual as compared to the zone nearer to the cylinder

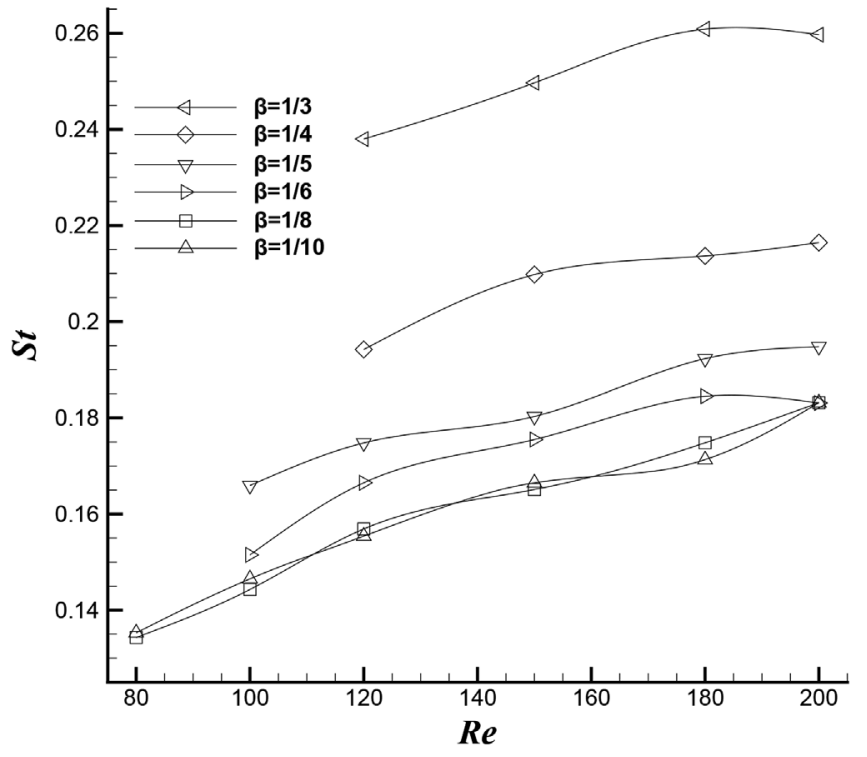

Fig. 8. The variation of $S t$ with Re for various $\beta$.

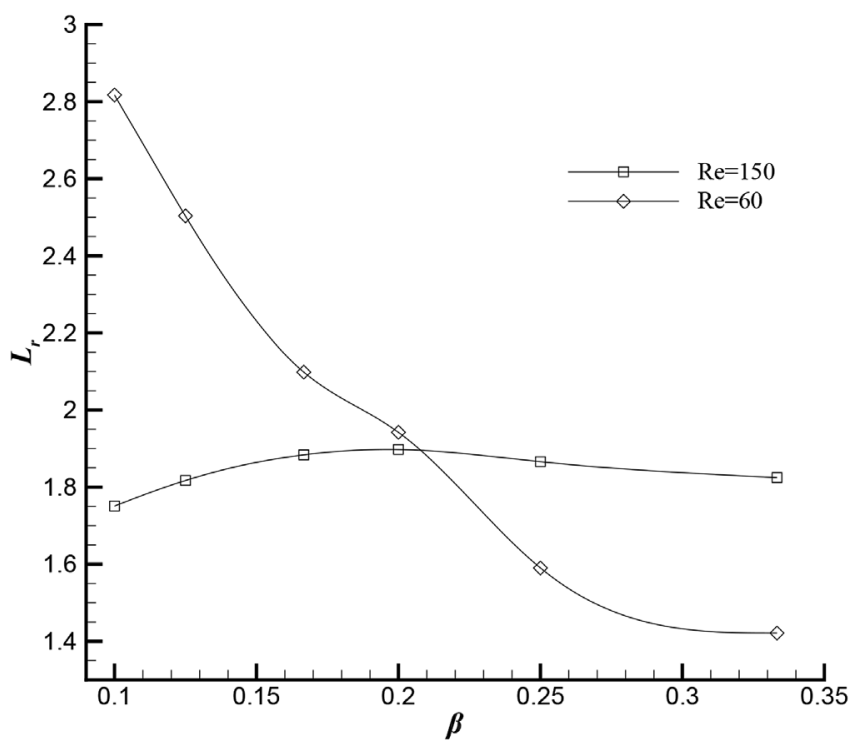

Fig. 9. The variation of recirculation length, $L_{r}$ as a function of blockage ratios $\beta$ for $\operatorname{Re}=60,150$.

surface. The variation in the vorticity flux far downstream from the cylinder is marginal and does not show any appreciable change. Near to the body, the maximum vorticity flux is experienced due to alternate formation of vortices during vortex shedding cycle. Near the walls, the flux is even higher as compared to near the body. This happens due to the confluence of the wall generated vorticity and the vorticity being generated from the cylinder.

\subsection{Heat transfer characteristics}

The forced convective heat transfer characteristics have been explored by the quantitative values of the Nusselt number, delineating the amount of heat transfer from the cylinder surface to free stream. Fig. 11 a and b show the variation of the local Nusselt number $N u_{l}$ over the cylinder surface for various values of the Reynolds number and at $\beta=1 / 3$ (Fig. 11a), $\beta=1 / 6$ (Fig. 11b). The local Nusselt number attains its maximum value at the front stagnation region and decreases gradually over the cylinder surface. From the heat balance equation at the cylinder wall, it can easily be shown that the local value of the Nusselt 


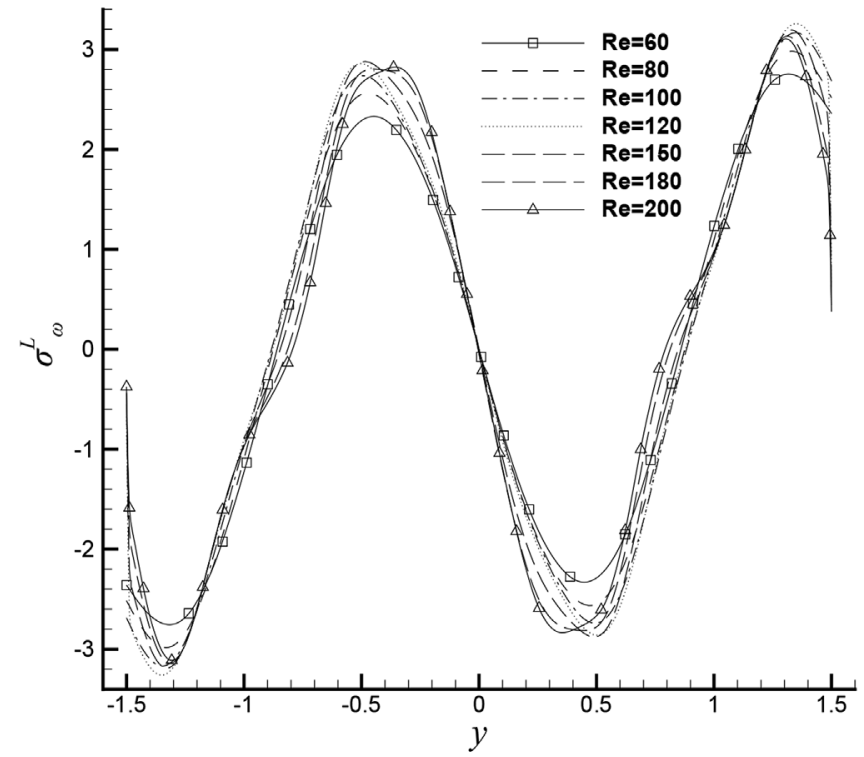

(a)

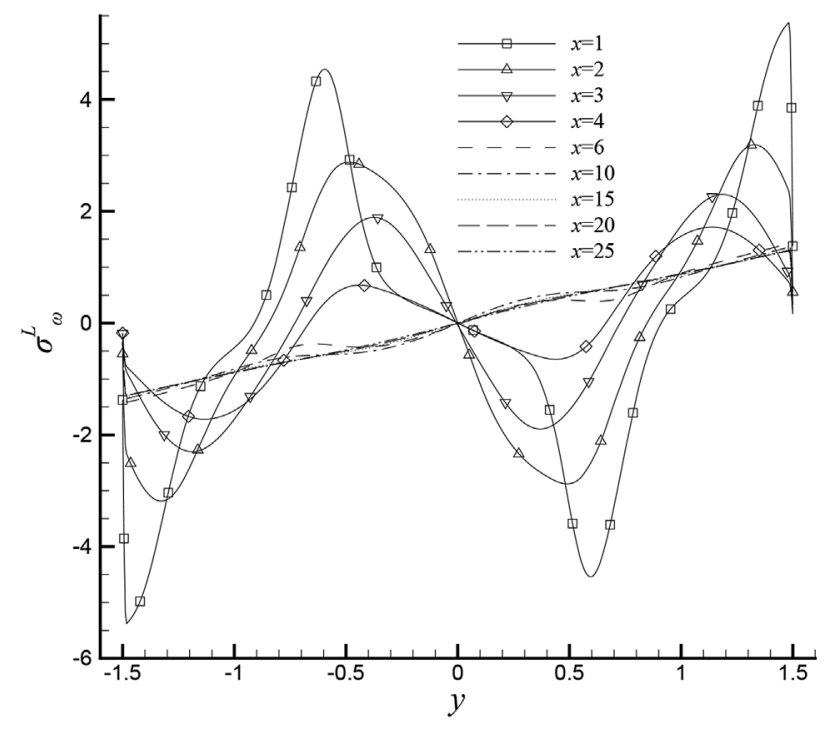

(b)

Fig. 10. The variation of $\sigma^{L} \omega$ (a) for various Re, along the vertical cross section at $x=2$ and at $\beta=1 / 3$, (b) across various cross sectional planes for $\operatorname{Re}=150$

number is dictated by the local temperature gradient (also from Eq. (11a)). This is quite apparent, as there is a maximum crowding of temperature contours at the front stagnation region thereby reducing the thermal boundary layer thickness. Reduction of thermal boundary layer results in higher temperature gradient. In the singularity points (at the sharp corners of the cylinder) the Nusselt number goes through a sudden jump in its magnitude, before reducing to its minimum value at the back stagnation region of the cylinder. On comparison between Fig. 11a-b, it can be observed that the maximum value of the local Nusselt number increases with increase in confinement. For a particular confinement as the Reynolds number is increased, the value of local Nusselt number also increases [25].

The variation of average Nusselt number, $N u_{\text {avg }}$ as a function of $\mathrm{Re}$ and for various values of $\beta$, is shown in Fig. 12. The average Nusselt

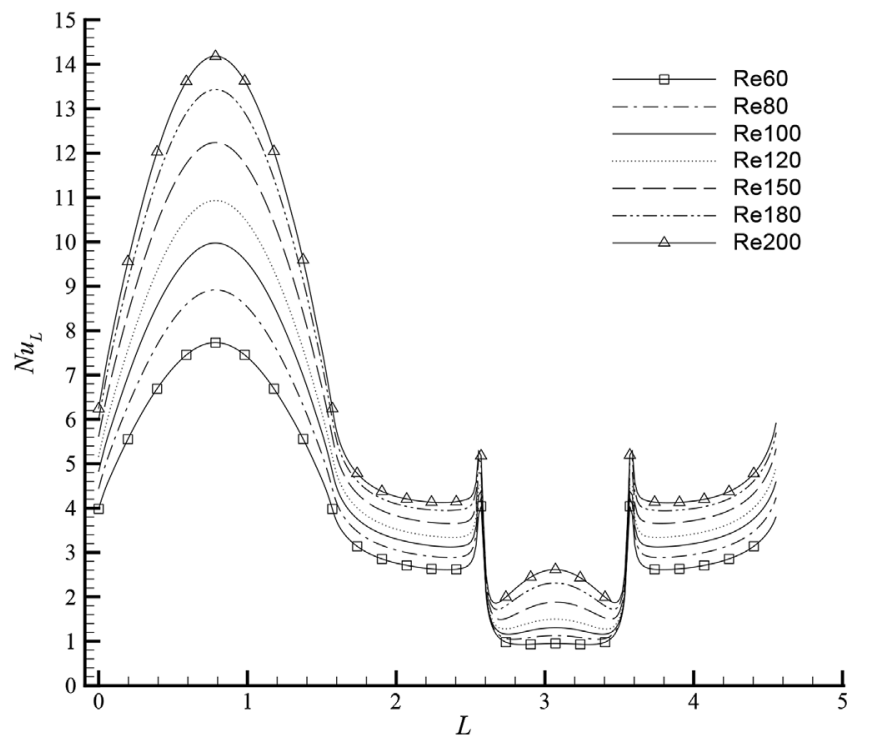

(a)

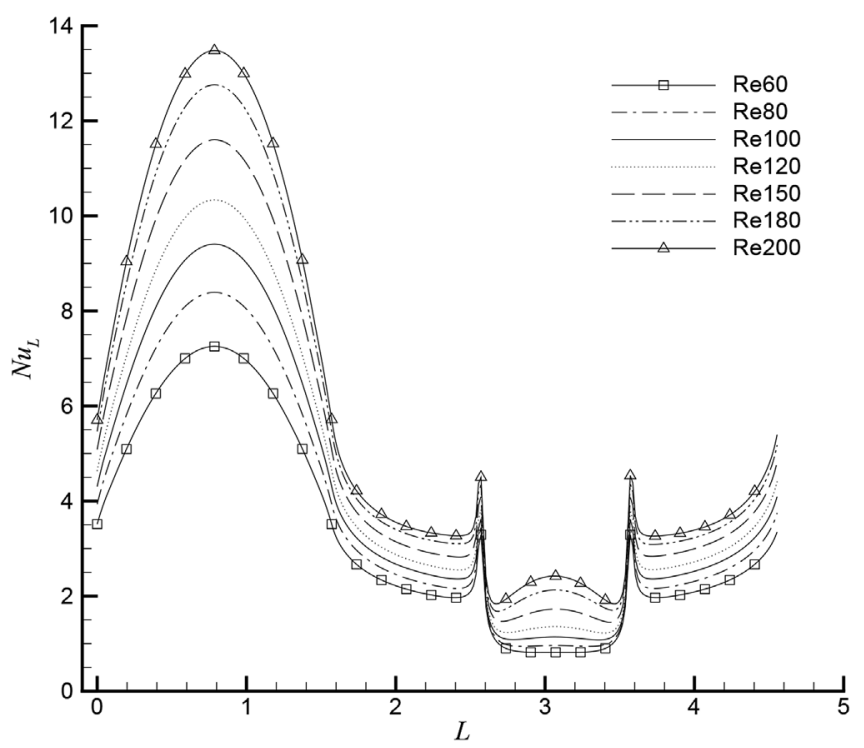

(b)

Fig. 11. The variation of the local Nusselt number $N u_{l}$ over the cylinder surface for various values of the Reynolds number and at (a) $\beta=1 / 3$; (b) $\beta=1 / 6$.

number is calculated by time averaging the spatially averaged local Nusselt number over the cylinder surface. As shown by the figure, increasing Reynolds number increases $N u_{\text {avg }}$. On other hand, for a constant Reynolds number, the $\mathrm{Nu}$ avg increases for increase in confinement. Analogous to that of the Strouhal number variation, it is seen that at lower values of $\beta$, the relative difference in the magnitude of the Nusselt number is less for the range of Reynolds numbers considered in the present investigation. Increase in heat transfer on increasing blockage ratio can be attributed to the increase in Strouhal number. The heat is advected faster from the cylinder surface and thereby increasing the heat transfer.

\section{Conclusions}

A numerical investigation is carried out to analyse the effect of confinement on fluid flow and heat transfer characteristics past a blunt 


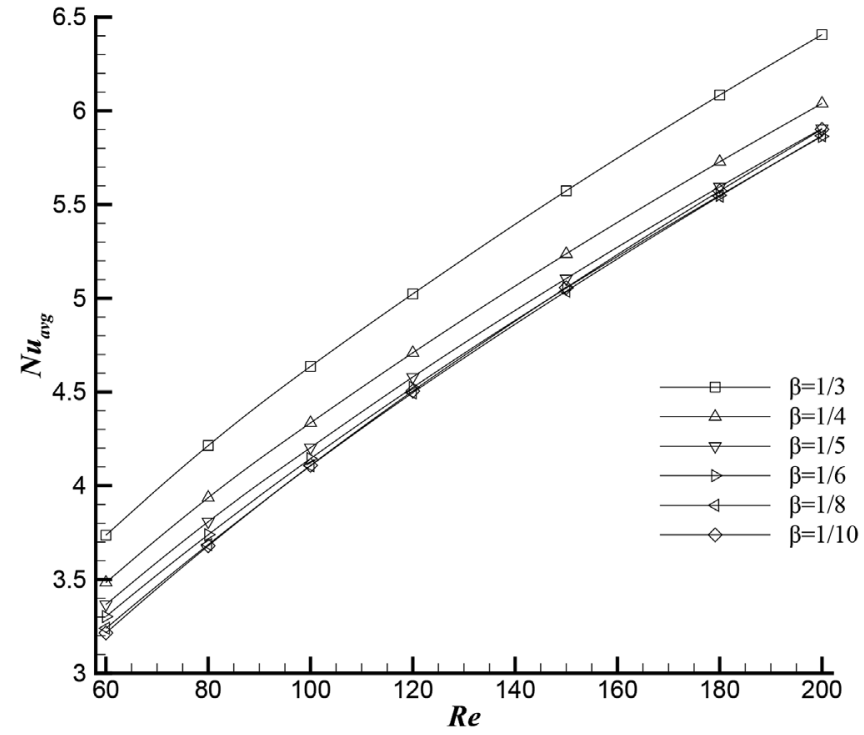

Fig. 12. Variation of $N u_{\text {avg }}$ as a function of Re and at various $\beta$.

headed cylinder. Utilizing air $(\mathrm{Pr}=0.71)$ as an operating fluid, flow simulations have been carried out for various ranges of channel confinements $1 / 3 \geq \beta \geq 1 / 10$ and the Reynolds numbers (based on projected length of the cylinder) $60 \leq \operatorname{Re} \leq 200$.

The simulation results exhibit the following conclusions:

- Increase in the Reynolds number decreases the drag coefficient. The value of the drag force acting on both the walls (upper and bottom) are same in magnitude. The magnitude of the drag coefficient increases as the value of $\beta$ increases.

- For a constant Re, the value of $C_{L, r m s}$ increases with decrease in $\beta$.

- For a constant Re, the value of $S t$ increases with increase in $\beta$. For a constant $\beta$, the value of Strouhal number increases with increase in Re.

- For smaller channel height and at low Re, the wake separation region is shorter in length as compared to the larger channels. On the other hand, for higher Reynolds Number, the recirculation length is almost constant with increasing $\beta$.

- The magnitude of vorticity flux increases with the increase in Re. The variation of $\sigma^{L}{ }_{\omega}$ across various cross sections found to at its maximum strength nearer to the cylinder.

- The local Nusselt number attains its maximum value at the front stagnation region and decreases gradually over the cylinder surface and its maximum magnitude is found to be increasing with enhancing the magnitude of $\beta$. Increasing Reynolds number, increases the value of the local Nusselt number.

- As Reynolds number is increasing the $N u_{\text {avg }}$ also increases for a constant confinement height. For a constant Reynolds number, the $\mathrm{Nu} u_{\text {avg }}$ also increases for increase in blockage ratios.

\section{References}

[1] R.W. Davis, E.F. Moore, L.P. Purtell, A numerical and experimental study of confined flow around rectangular cylinders, Phys Fluids 27 (1984) 46-59.

[2] H. Suzuki, Y. Inoue, T. Nishimura, K. Fukutani, K. Suzuki, Unsteady flow in a channel obstructed by a square rod (crisscross motion of vortex), Int J Heat Fluid Flow 14 (1993) 2-9.

[3] A. Sohankar, C. Norberg, L. Davidson, Numerical simulation of unsteady low-reynolds number flow around rectangular cylinders at incidence, J Wind Engg Ind Aerodyn 69 (1997) 189-201.

[4] M. Breuer, J. Bernsdorf, T. Zeiser, F. Durst, Accurate computations of the laminar flow past a square cylinder based on two different methods: Lattice-Boltzmann and finite-volume, Int J Heat Fluid Flow 21 (2000) 186-196.

[5] H. Abbassi, S. Turki, S.B. Nasrallah, Numerical investigation of forced convection in a plane channel with a built-in triangular prism, Int J Therm Sci 40 (2001) 649-658.

[6] J. Chakraborty, N. Verma, R.P. Chhabra, Wall effects in flow past a circular cylinder in a plane channel, Chem Engg Process 43 (2004) 1529-1537.

[7] A. Sharma, V. Eswaran, Effect of channel confinement on the two-dimensional laminar flow and heat transfer across a square cylinder, Num Heat Transf Part A 47 (2005) 79-107.

[8] M. Sahin, R.G. Owens, A numerical investigation of wall effects up to high blockage ratios on two-dimensional flow past a confined circular cylinder, Phys Fluids 16 (2004) 1305.

[9] A.K. Dhiman, R.P. Chhabra, V. Eswaran, Flow and heat transfer across a confined square cylinder in the steady flow regime: effect of Peclet number, Int J Heat Mass Transf 48 (2005) 4598-4614.

[10] A.K. Dhiman, R.P. Chhabra, V. Eswaran, Steady flow across a confined square cylinder: effects of power-law index and blockage ratio, J Newt Fluid Mech 148 (2008) 141-150.

[11] M. Rahnama, H.H. Moghaddam, Numerical investigation of convective heat transfer in unsteady laminar flow over a square cylinder in a channel, Heat Transf Engg 26 (2005) 21-29.

[12] P.P. Patil, S. Tiwari, Effect of blockage ratio on wake transition for flow past square cylinder, Fluid Dyn Res 40 (2008) 753-778.

[13] A. Sohankar, Large eddy simulation of flow past rectangular-section cylinders: side ratio effects, J Wind Engg Ind Aerodyn 96 (2008) 640-655.

[14] B. Kumar, S. Mittal, Effect of blockage on critical parameters for flow past a circular cylinder, Int J Numer Meth Fluids 50 (2006) 987-1001.

[15] S. Mettu, N. Verma, R.P. Chhabra, Momentum and heat transfer from an asymmetrically confined circular cylinder in a plane channel, Heat Mass Transf 42 (2006) 1037-1048.

[16] S. Singha, K.P. Sinhamahapatra, Flow past a circular cylinder between parallel walls at low Reynolds number, Ocean Engg 37 (2010) 757-769.

[17] A.K. De, A. Dalal, Numerical study of laminar forced convection fluid flow and heat transfer from a triangular cylinder placed in a channel, J Heat Transf 129 (2007) 646-656.

[18] S. Srikanth, A.K. Dhiman, S. Bijjam, Confined flow and heat transfer across a triangular cylinder in a channel, Int J Therm Sci 49 (2010) 2191-2200.

[19] J.L. Rosales, A. Ortega, J.A.C. Humphrey, A numerical simulation of the convective heat transfer in confined channel flow past square cylinders: comparison of inline and offset tandem pairs, Int J Heat Mass Transf 44 (2001) 587-603.

[20] J.P. Dulhani, S. Sarkar, A. Dalal, Effect of angle of incidence on mixed convective wave dynamics and heat transfer past a square cylinder in cross flow at $R e=100$, In J Heat Mass Transf 74 (2014) 319-332.

[21] FLUENT 14.0 User's guide, vol. 5, Fluent Inc., Lebanon, NH, 2001.

[22] GAMBIT 2.2 User's guide, Fluent Inc., Lebanon, NH, 2004.

[23] C.H.K. Williamson, Vortex dynamics in the cylinder wake, Annu Rev Fluid Mech 28 (1996) 477-539.

[24] E.R.G. Eckert, E. Soehngen, Distribution of heat transfer coefficients around circular cylinders in crossflow at Reynolds numbers from 20-500, Trans ASME 74 (1952) 343-347.

[25] G. Biswas, S. Sarkar, Effect of thermal buoyancy on vortex shedding past a circular cylinder in cross flow at low Reynolds numbers, Int J Heat Mass Transf 52 (2009) 1897-1912.

[26] S. Sarkar, A. Dalal, G. Biswas, Unsteady wake dynamics and heat transfer in forced and mixed convection past a circular cylinder in cross flow for high Prandtl numbers, Int J Heat Mass Transf 54 (2011) 3536-3551.

[27] S. Sarkar, A. Dalal, G. Biswas, Mixed convective heat transfer from two identical square cylinders in cross flow at $\mathrm{Re}=100$, Int J Heat Mass Transf 53 (2010) 2628-2642.

[28] S. Sarkar, S. Ganguly, A. Dalal, Buoyancy driven flow and heat transfer of nanofluids past a square cylinder in vertically upward flow, Int J Heat Mass Transf 59 (2013) 433-450. 